Sociologie et sociétés

\title{
Modèles qualitatifs, modèles quantitatifs dans la connaissance scientifique
}

\section{Gilles G. Granger}

Volume 14, numéro 1, avril 1982

La sociologie : une question de méthode?

Sociology: A Matter of Methods?

URI : https://id.erudit.org/iderudit/006768ar

DOI : https://doi.org/10.7202/006768ar

Aller au sommaire du numéro

Éditeur(s)

Les Presses de l'Université de Montréal

ISSN

0038-030X (imprimé)

1492-1375 (numérique)

Découvrir la revue

Citer cet article

Granger, G. G. (1982). Modèles qualitatifs, modèles quantitatifs dans la connaissance scientifique. Sociologie et sociétés, 14(1), 7-13.

https://doi.org/10.7202/006768ar
Résumé de l'article

L'évolution de la prise de conscience de la nature profonde de la pensée scientifique pourrait être symbolisée, très schématiquement, par trois devises, dont chacune réinterprète d'une certaine manière et rectifie la précédente. On a d'abord proclamé qu'il n'y avait de science que de l'universel; puis, qu'il n'y avait de science que du mesurable. Nous devrions dire aujourd'hui : il n'y a de science que du structurable. Profession de foi qui ne récuse nullement les deux précédentes, mais les relativise, et donne un sens nouveau à l'universel et au mesurable. C'est de cette manière qu'il conviendrait, me semble-t-il, de reconnaître le rôle et la place des modèles qualitatifs dans la pensée scientifique. 


\section{Modèles qualitatifs, modèles quantitatifs dans la connaissance scientifique*}

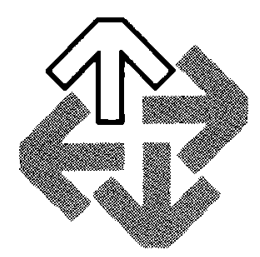

GILLES G. GRANGER

C'est souvent avec l'intention d'en restreindre la portée que l'on dit d'un modèle qu'il est qualitatif. L'adjectif est supposé excuser par avance les incertitudes d'une pensée vague et encore insuffisamment rationnelle. Est-ce ainsi qu'il faut l'entendre? Je voudrais essayer de caractériser brièvement le statut et la fonction des modèles qualitatifs dans la science, et d'en défendre l'idée, à la fois contre ceux qui n'y veulent voir que des ébauches de pensée, et contre ceux qui en saluent l'avènement comme une victoire de l'irrationnel.

\section{QU'APPELONS-NOUS «MODĖLE»?}

Nous donnerons ici au mot «modèle» une acception sans doute assez conforme à l'usage, mais qu'il convient pourtant de préciser. Un modèle sera pour nous une représentation abstraite des phénomènes; même s'il s'agit d'une réalisation matérielle, ce qui importe dans le modèle ainsi entendu, c'est sa fonction de schématisation; le but n'est pas de reproduire en quelque manière le phénomène, mais de le représenter en manifestant l'opposition d'une forme à un contenu. Malgré les apparences contraires, les modèles du logicien, qui sont en un sens plus «concrets» que ce dont ils sont des modèles, obtiennent dans une autre perspective le même résultat qui est de rendre saisissable le rapport opératoire d'une forme à un contenu.

* Ce texte est issu d'une Communication faite au Convegno: Il sapere come rete di Modelli (Modena, Italie, 20-23 janvier 1981). Il est paru en traduction italienne dans $I l$ sapere come rete di modelli. La conoscenza oggi, Edizione Janini, 1981. Je remercie les organisateurs du Convegno d'en autoriser la publication en français. 
En ce qui concerne les modèles des sciences empiriques qui nous occupent ici, nous ferons tout d'abord deux remarques. En premier lieu, nous soulignerons que la différence ici faite entre modèle et théorie ne joue pas un rôle essentiel. Une théorie sera considérée seulement comme un modèle plus ample et plus ambitieux. Un modèle au sens strict s'en distinguera parce qu'il est plus local (en un sens évidemment plus large que le seul sens spatial), et parce que les principes ou présupposés dont il part sont plus spécifiques, plus immédiatement révisables en fonction de résultats expérimentaux. Différence de degré, donc, plutôt que de nature. En second lieu, nous observerons qu'il y a lieu de reconnaître comme également dignes du titre deux espèces assez généralement irréductibles de modèles, que nous désignerons respectivement comme modèles-machines et comme modèles-systèmes. Les premiers visent à représenter un processus, et décrivent à proprement parler un fonctionnement; les seconds décrivent statiquement une structure, comme interrelation d'éléments: un système phonologique, une classification zoologique sont à cet égard des modèles.

Ces remarques générales appelleraient sans doute une discussion où nous ne saurions maintenant nous engager; on demandera donc seulement de les considérer comme délimitant conventionnellement le champ des réflexions qui vont suivre touchant le rôle et la fonction de modèles qualitatifs, par opposition aux modèles quantitatifs auxquels le développement des sciences de la nature nous a depuis longtemps habitués.

\section{LE QUALITATIF ET LE QUANTITATIF}

Qu'est-ce qu'un modèle quantitatif? On sait depuis Leibniz de façon tout à fait claire que la mathématique n'est pas seulement ni même essentiellement science de la quantité. L'utilisation de la mathématique dans un modèle ne saurait donc le caractériser comme quantitatif. Mais non plus la seule présence du nombre, comme il apparaîtra plus avant. Un modèle est quantitatif quand on y fait usage de la mesure. Mesurer, au sens large où nous l'entendrons, c'est faire correspondre aux opérations de calcul effectuées dans un corps de nombres, des opérations empiriques bien définies pour le phénomène considéré. Sans doute est-il permis d'affaiblir plus ou moins cette exigence et d'admettre que les nombres mesurants n'ont que les propriétés d'un anneau, voire d'une structure plus faible encore. On pourra' définir ainsi une hiérarchie de quantifications plus ou moins parfaites, et préciser à ces différents niveaux les conditions mathématiques de la mesure. Mais la construction de modèles quantitatifs exige encore que soient explicitées des conditions sémantiques de la mesure, que soient indiqués, dans chaque cas le sens opératoire empirique des expressions: "discerner», "ordonner», «ajouter» des événements. Les difficultés soulevées dès le milieu $\mathrm{du} \mathrm{XIX}^{\mathrm{e}}$ siècle par les premières tentatives de psychophysique ont montré l'importance fondamentale, aujourd'hui encore, pourtant, insuffisamment reconnues, de ces conditions préalables à tout essai de modèles qualitatifs, singulièrement dans les sciences de l'homme. Or les événements nous sont d'abord immédiatement donnés comme qualités.

Sans entrer plus avant dans les problèmes philosophiques posés par la saisie de la qualité, nous nous contenterons de distinguer deux niveaux dans cette saisie. La qualité est d'abord, sans doute, un vécu absolu et unique, que la langue naturelle nous permet, peut-être, dans une certaine mesure de transmettre et les différents arts de recréer, mais que la science ne saurait viser à décrire comme tel. Mais elle est aussi vécue au niveau de la forme, et c'est ce vécu formel que la connaissance scientifique vise à transformer en concepts.

Une forme est, me semble-t-il, appréhendée à la fois comme contraste et comme continuité. En tant que contraste, elle est partition du champ perceptif, elle est découpage. La notion de "flou» est à cet égard digne d'attirer notre attention, car elle sous-tend l'interférence de deux échelles de perception, et l'oscillation entre deux acco- 
modations de la visée: au macro-niveau, le contraste est net et la forme prégnante, au micro-niveau, le contraste s'atténue et la forme tend à se dissoudre. Mais le contraste ne suffirait pas à l'apparition de la forme; il lui faut encore la continuité. Nous nous plaçons naturellement ici sur un plan antérieur à celui de la conceptualisation du continu, dont l'histoire se confond pour une bonne part avec celle de la mathématique depuis les Grecs jusqu'aux géomètres de la fin du dernier siècle. Antérieurement à cette conceptualisation, la notion de continuité ne peut qu'être commentée par d'autres intuitions: celle d' "unité», celle de l'«impossibilité d'une coupure»... Elle se fixe essentiellement au moyen d'images spatiales, et l'on pourrait être tenté de croire qu'elle est caractéristique de la catégorie de spatialité, auquel cas on serait quasiment contraint de reconnaître qu'il n'est de formes, après tout, que spatiales. Sans doute la spatialité a-t-elle pour nous un privilège biologique qui se marque apparemment dans toutes les langues naturelles. Il me semble pourtant qu'une notion comme celle de continuité est de nature pour ainsi dire pré-spatiale, plus profonde, et qu'elle est susceptible de caractériser des formes pour lesquelles la spatialité n'est qu'un mode de représentation et nullement une condition essentielle de subsistance.

C'est en vain que l'on s'interrogerait sur la priorité du continu sur le discontinu, une fois reconnue leur inséparabilité dans la saisie de la forme. Dans la perception, on ne saurait dire que la continuité précède le discontinu, car elle en est corrélative. D'un point de vue plus radical, pour le philosophe qui voudrait mettre au jour les conditions de possibilité de la connaissance, la réponse est donnée par Kant dans l'Analytique transcendantale, qui situe sur le même plan, comme principes "mathématiques" de l'entendement, les «axiomes de l'intuition» et les «anticipations de la perception». Les premiers énoncent que «toute intuition est une grandeur extensive», proposition dont le sens minimal est que tout ce qui peut être perçu est découpable en parties, saisissables dans leur discontinuité. Les seconds posent que «dans tout phénomène le réel, qui est objet de la sensation, a une grandeur intensive ", c'est-à-dire «qui n'est appréhendée que comme unité, et dans laquelle la pluralité ne peut être représentée que dans son mouvement d'approche du degré zéro", durch Annäherung zur Negation $=0($ Kritik der r.V.B. 210). Une telle caractérisation de l'intensif sous-tend évidemment la notion de continuité.

Ainsi, c'est à la fois en tant que contraste et continuité, sous les espèces de la forme, que la qualité peut être déterminée, et si des modèles qualitatifs sont possibles pour la science, c'est par une conceptualisation de la forme.

\section{LA CONCEPTUALISATION DE LA FORME}

Le premier pas vers une conceptualisation de la forme c'est assurément l'invention d'une procédure de description canonique. C'est ce qui peut être obtenu par réduction de la forme dans un canevas binaire. Telle cellule du canevas, ou tel point, appartient ou n'appartient pas à la forme qu'il faut décrire. On perd ainsi bien évidemment l'un des moments constitutifs de la forme, qui est sa continuité. Mais le choix de la dimension et de la configuration des mailles du réseau permet de contrôler le flou de la représentation et d'imiter ainsi le flou de l'expérience même. Ce filtre conventionnel délimite les zones où règne la continuité intuitive et en précise le seuil. Son adéquation constitue déjà une connaissance relative à la forme décrite, bien qu'on doive reconnaître qu'à ce niveau celle-ci demeure tout à fait incomprise.

On observera que le nombre s'introduit ici assez naturellement comme instrument de repérage, par exemple dans la perspective cartésienne de représentation des courbes ou des surfaces. La continuité intuitive peut alors être retrouvée par le détour d'une mesure utilisant, d'abord fort confusément, des suites supposées denses de nombres. Mais la métrique n'est pourtant pas essentielle à cette représentation des formes, bien que ce préjugé tenace ait joué un rôle irremplaçable dans la création et le développement 
de l'Analyse. Un géomètre de génie, né deux siècles trop tôt, avait pourtant dès le temps de Descartes, compris que l'on pouvait rendre indépendants de la mesure la description et l'engendrement des formes: c'est Girard Desargues jetant les bases de la géométrie projective. Et Leibniz lui-même, quoique son invention du Calcul infinitésimal prolonge la représentation cartésienne, inspiré par une philosophie où la continuité joue un rôle fondamental, tentait obstinément déjà d'édifier une théorie non métrique des formes.

Ce n'est que plus tard, néanmoins, qu'un pas décisif sera fait vers la seconde caractérisation de la forme. Il ne s'agit plus seulement de décrire; mais de comprendre. La forme est alors conceptualisée comme invariant d'un système de transformations. Certes, l'intuition sensible y perd, et ce que nos sens distinguent, nos concepts sont souvent impuissants à le dissocier en fait: le mathématicien ne sait formuler le groupe de transformations correspondant ${ }^{1}$. Mais un sens opératoire est désormais donné, en principe: à la notion de continuité de la forme, et celle-ci est appréhendée globalement par concepts. De véritables modèles qualitatifs, importants dans l'histoire des sciences de la nature, paraissent se rattacher directement à cette étape de la conceptualisation de la forme. Le cas exemplaire est sans doute fourni par la cristallographie. Les modèles de structure des cristaux établis par Bravais, puis Sohnke, Schönflies, Fedorov expliquent des formes naturelles à partir des invariants nécessairement associés à des groupes de transformation dans l'espace tridimensionnel. Bel exemple de modèles-systèmes, puisqu'ils n'ont pas pour objet des processus, et qui sont bien des modèles qualitatifs, $n$ 'introduisant de manière essentielle aucune mesure. Sans doute des nombres apparaissentils, dans la caractérisation des 230 groupes de position, des 14 réseaux cristallins; mais ces nombres ne produisent pas de quantification, ce sont des index d'opérations, comme il en apparaît toujours en théorie des groupes. Il est bon peut-être d'insister sur cette distinction triviale, pour bien montrer qu'un modèle qualitatif n'est pas nécessairement un modèle sans nombres, mais seulement un modèle sans mesure. On aimerait pouvoir citer d'aussi beaux succès de cette conceptualisation de la forme dans les sciences de l'homme. Mais la détermination suffisamment précise de transformations est alors incomparablement plus difficile dans le cas des comportements humains que dans l'univers aisément abstrait des relations spatiales; des tentatives ont été développées cependant pour donner de cette manière un sens conceptuel à certaines formes de l'objet humain, tels les essais de Jean Piaget sur la psychologie de l'intelligence.

Reste un troisième mode de conceptualisation de la forme qui peut servir de fondement à des types nouveaux de modèles qualitatifs. La forme est alors caractérisée par ses singularités. On prendra ici ce mot en un sens plus général que son sens technique en Analyse : un extremum, un point d'inflexion seront comptés au nombre des singularités. Il semble que l'on abandonne en ce cas la détermination globale d'une forme pour s'en tenir à ses accidents locaux. Mais dans nombre de cas, pour la description et l'explication des phénomènes, ce sont ces accidents qui importent: tant il est vrai que dans la saisie de la forme l'intuition de la continuité est inséparable de la rencontre des ruptures. Par ailleurs, le point de vue global réapparaît dans la perspective imaginée par R. Thom avec sa théorie des catastrophes. Dans le cas où la forme d'un phénomène - la loi de covariation des facteurs qui le décrivent dans un espace convenable - dépend elle-même d'une liaison entre un petit nombre de paramètres, les mutations de forme du phénomène correspondent aux singularités de cette liaison dans l'espace des paramètres. La distribution de celles-ci engendre des figures typiques, caractéristiques des changements de régime du phénomène. Un modèle ainsi conçu comporte donc deux étapes pour ainsi dire superposées, dont l'une décrit le jeu de varia-

1. Cette remarque de René Thom n'est pourtant pas une objection dirimante contre la valeur de principe d'une telle conceptualisation de la forme. 
bles stratégiques l'autre celui des variables «dynamiques» réglant l'évolution du phénomène dans les limites d'un certain champ de stabilité. Sans aucunement exclure les considérations quantitatives, en particulier au niveau de cette évolution dynamique, le modèle est essentiellement qualitatif en ce qu'il fournit les conditions de cette stabilité et repère les mutations radicales de formes. La connaissance de la forme, de purement descriptive, tend ici à devenir génétique. Non pas simplement au sens usuel de l'enchaînement nécessaire d'états successifs à partir d'un état initial; mais au sens plus profond qui permettrait de dire pourquoi telle forme se produit et se maintient plutôt que telle autre, et dans quelles conditions telle forme disparaît.

On pourrait se demander si le développement - effectif ou en projet - de modèles qualitatifs fait apparaître la prédominance de l'usage d'un outil mathématique privilégié. Sans doute peut-on citer comme candidats tout désignés: les calculs logiques, l'Algèbre comme théorie la plus générale des systèmes de combinaison d'opérations quelconques, la Topologie comme théorie par excellence des configurations d'objets. Mais la réalité mathématique - je veux dire l'usage réel de l'outil mathématique - s'accommode rarement de distinctions aussi tranchées, et l'instrumentation la plus efficace est souvent la plus composite, pour peu qu'elle ne procède pas d'un goût immodéré de la sophistication et de l'artifice, mais bien d'un souci d'adaptation à l'objet. Par ailleurs, l'important n'est peut-être pas tant ici la nature formelle de l'outil que la manière de l'utiliser et l'esprit dans lequel on l'utilise. Des concepts mathématiques supposant la mesure de certaines variables peuvent parfaitement apparaitre, à titre d'auxiliaires dans un modèle qui restera qualitatif si sa visée essentielle est la détermination d'une forme. En outre, la connaissance conceptuelle des formes n'implique en aucune manière un renoncement systématique et définitif à une connaissance quantitative; certes, elle ne saurait être considérée a priori comme un pis-aller provisoire ; mais, dans les cas les plus favorables, quand la visée d'une connaissance quantitative est possible, elle en le sera le complément essentiel, en lui fournissant un cadre et en lui donnant un sens.

\section{VALEUR EXPLICATIVE ET EFFICACITÉ DES MODĖLES QUALITATIFS}

Les trois modes de traitement conceptuel de la forme que nous ayons tenté de définir, bien qu'ils puissent et doivent sans doute concourir simultanément à l'édification de modèles qualitatifs, s'ordonnent selon une hiérarchie conventionnellement notée par ces trois mots : décrire, comprendre, expliquer. Expliquer, c'est à dire, ayant repéré un phénomène comme totalité et dissocié ses parties (c'est «décrire»), ayant établi les relations et les contraintes qui les associent (c'est «comprendre»), savoir insérer ce système dans un système plus vaste dont dépend sa genèse, sa stabilité et son déclin. Ainsi définie comme la forme la plus complète de la connaissance scientifique, l'explication est, bien entendu, toujours relative et provisoire. En termes très généraux, qui resteraient à définir dans chaque cas d'espèce, on pourrait dire qu'elle consiste en fin de compte à préciser dans un phénomène les relations du «local» et du «global», et l'on voit alors qu'en ce sens elle ne saurait se passer de considérer des formes. Le rôle des modèles qualitatifs est donc éminent. Dans les sciences humaines, en particulier, là où l'introduction de la mesure ne peut être légitimée qu'au prix d'un immense effort d'analyse conceptuelle, la recherche d'un modèle qualitatif peut être un préalable indispensable à toute objectivation du phénomène, aussi bien qu'elle peut être le but ultime de l'explication. Un exemple modeste, mais particulièrement significatif, d'explication par un modèle qualitatif, nous serait donné par l'hypothèse de transformation du système phonologique vocalique dans un patois savoyard présenté naguère par $\mathrm{A}$. Martinet (Économie des changements phonétiques, 1955). À partir d'un système postulé, instable, l'auteur montre le passage à des formes stabilisées, dans lesquelles les modifications locales ont entraîné un remaniement global conduisant à l'état actuel. Il s'agit 
bien là, sous réserve d'un contrôle de la description phonologique, d'une tentative d'explication par le moyen d'une statique comparative purement formelle.

D'une certaine manière, reconnaître l'importance des modèles qualitatifs c'est échapper aux artificielles contraintes d'un postivisme dogmatique, tout en continuant d'accepter les exigences raisonnables d'un positivisme bien-tempéré. Le modèle qualitatif, en effet, répond à un désir de rechercher «la raison des effets», sans pour autant avoir recours à des entités métaphysiques que, par leur nature, aucune épreuve ne saurait disqualifier. Et la visée du "global" qui est inhérente à tout modèle qualitatif ne se confond jamais avec celle d'une totalité inconditionnée et d'un fondement ultime, qui hante nécessairement et légitimement le philosophe, mais qu'aucune pensée scientifique ne peut embrasser sans perdre irrémédiablement tout son sens et toute sa vertu.

Mais tout modèle qualitatif n'est pas forcément significatif et efficace. À quelles conditions peut-il l'être? Il nous semble qu'il doive, en premier lieu, être assorti d'indications suffisamment précises sur l'échelle du phénomène à laquelle il est valable. Si la réduction conceptuelle d'une forme est, en tant que telle, indépendante de toute grandeur, la réalisation d'une forme dans le phénomène est au contraire, en général, conditionnée par sa taille. Le rapport du quantitatif au qualitatif fait apparaître, à cet égard une sorte de cercle, s'il est vrai que le modèle qualitatif est un cadre d'interprétation pour les mesures, et qu'en revanche la validité du modèle qualitatif est délimitée par un ordre de grandeur. Mais de telles antinomies ne sont qu'apparentes. La pensée scientifique active ne consiste pas à partir d'abord d'une connaissance fondée, mais d'une connaissance provisoire; l'application du modèle à un domaine délimité par des mesures grossières permet de raffiner celles-ci et de mieux déterminer le champ de validité du modèle, un peu comme la réinjection dans un algorithme d'une valeur approchée déjà obtenue par le moyen de cet algorithme permet de calculer une valeur de plus en plus exacte. Bien entendu, de même que dans cette métaphore mathématique la convergence de la procédure n'est nullement acquise de soi, de même l'aptitude du modèle qualitatif à permettre d'améliorer la détermination de son champ est-elle une propriété non-triviale et l'un des signes de sa valeur objective.

En second lieu, le modèle qualitatif ne saurait être efficace s'il ne fournit le moyen de repérer par des critères expérimentaux les facteurs critiques de détermination de la forme, que nous dénommions plus haut facteurs «stratégiques». Qu'ils puissent se présenter comme des "variables cachées" par opposition aux variables plus manifestes qui définissent le fonctionnement ou la structure du phénomène lui-même, voilà qui ne facilite pas la tâche, et peut donner carrière à une imagination libérée du contrôle empirique. Ici encore, des conjectures de quantification plus ou moins fortes sont le plus souvent requises. Par où l'on voit, finalement, qu'un modèle n'est pas qualitatif parce qu'il serait étranger à toute considération de grandeurs, mais parce qu'il prend celles-ci comme moyen et subordonne la détermination des quantités à la détermination des formes. Et si tout modèle scientifique intéressant comporte une interprétation qualitative, il n'est guère douteux que tout modèle scientifique utilisable comporte des repères quantitatifs.

L'évolution de la prise de conscience de la nature profonde de la pensée scientifique pourrait être symbolisée, très schématiquement, par trois devises, dont chacune réinterprète d'une certaine manière et rectifie la précédente. On a d'abord proclamé qu'il n'y avait de science que de l'universel; puis, qu'il n'y avait de science que du mesurable. Nous devrions dire aujourd'hui: il n'y a de science que du structurable. Profession de foi qui ne récuse nullement les deux précédentes, mais les relativise, et donne un sens nouveau à l'universel et au mesurable. C'est de cette manière qu'il conviendrait, me semble-t-il, de reconnaître le rôle et la place des modèles qualitatifs dans la pensée scientifique. 


\section{RÉSUMÉ}

L'évolution de la prise de conscience de la nature profonde de la pensée scientifique pourrait être symbolisée, très schématiquement, par trois devises, dont chacune réinterprète d'une certaine manière et rectifie la précédente. On a d'abord proclamé qu'il n'y avait de science que de l'universel; puis, qu'il n'y avait de science que du mesurable. Nous devrions dire aujourd'hui : il n'y a de science que du structurable. Profession de foi qui ne récuse nullement les deux précédentes, mais les relativise, et donne un sens nouveau à l'universel et au mesurable. C'est de cette manière qu'il conviendrait, me semble-t-il, de reconnaître le rôle et la place des modèles qualitatifs dans la pensée scientifique.

\section{SUMMARY}

The evolution which has taken place in the consciousness of the deepest nature of scientific thought could be symbolized, in a very simplified fashion, by three catchwords, each of which reinterprets and rectifies the preceding one. First, it was proclaimed that science was only that which is universal, then that it was only that which is measureable. Today, we would have to say that science is only that which is structurable a statement of faith which in no way challenges the preceding ones, but relativizes them, giving a new meaning to the universal and measureable. It is in this light that, in the author's opinion, the role and place of qualitative models in scientific thought should be seen.

\section{RESUMEN}

La evolución de la toma de conciencia de la naturaleza profunda del pensamiento científico podría ser simbolizada, muy esquemáticamente, por tres máximas, donde cada una reinterpreta de una cierta manera y rectifica las anteriores. Primero se proclamó que no habia ciencia que de lo universal, despues que no había ciencia que de lo que se podia medir. Hoy en dia se podria decir que no hay ciencia que de lo estructurable. Profesión de fé que no rechaza de ninguna manera las dos anteriores pero las matiza y da un nuevo sentido a lo universal y a lo que se puede medir. Es de esta manera, piensa el autor, que convendría reconocer el papel y el lugar de los modelos cualitativos en el pensamiento científico. 